\title{
Connection setup and traffic aggregation in IP- based Elastic Optical Networks (EON)
}

\author{
Wajdi Halabi \\ Dept. CS \& IT \\ University College of Science \& Technology (UCST) \\ Khan-Yonis, Gaza Strip, Palestine \\ w.halabi@ucst.edu.ps
}

\begin{abstract}
To overcome the challenges of the increased IP traffic volume on the Internet, the IP/MPLS-based fixed grid DWDM network structure was proposed, which makes the interaction between both the IP layer and the optical wavelength routed fixed DWDM layer applicable. However, the deployment of this architecture was not only expensive, but powerconsuming. The emerging optical Flexgrid networks technology is nowadays suggested to be the potential candidate for future optical transport networks. This is because it provides extremely flexible and spectrally effective resources utilisation. Therefore, it has attracted the attention of network operators, optical networks equipment manufacturers and the wider research and development society. In this research work, we propose a novel multilayer routing scheme for an IP/MPLS-based optical Flexgrid network, which is based on the protocol suite defined by the GMPLS architecture. Furthermore, we evaluate its behaviour in an online dynamic traffic scenario.
\end{abstract}

Keywords-Elastic Optical Networking (EON), Flexible grid, flexgrid, bitrate variable transponders, DWDM, IP/MPLS, IP/WDM, IP/EON, GMPLS.

\section{INTRODUCTION (HEADING 1)}

By 2022, the continual growth of global IP traffic will reach an annual run rate of 4.8 zettabytes per year, which is nearly 11 times more than all the IP traffic generated in 2012 (437 Exabyte) [1]. This will obviously present a great challenge for internet Service Providers (SPs). This continual growth in bandwidth demand has forced network operators and Service Providers (SP) to install higher bit rates (e.g. 40 $\mathrm{Gb} / \mathrm{s}$ and now $100 \mathrm{~Gb} / \mathrm{s}$ per wavelength) [2], but it is expected that the fixed-grid 50GHZ (IUT) wavelength will net satisfy the requirement for bit rates greater than $100 \mathrm{~Gb} / \mathrm{s}$. For each connection in wavelength-routed networks, a full allocation of a wavelength between source and destination nodes is required, even though the traffic is not enough to use all the available capacity.

This problem has revealed the deficiencies of the rigid DWDM networks when it comes to matching client layer requirements efficiently, hence the deployment of expensive IP/MPLS layers to aggregate traffic via its grooming functionality was proposed.

The emerging optical Flex-grid networks [3][4], which offer flexible and very effective utilisation of spectral resources, are potential candidates for future optical networks.

Compared to the ITU-T DWDM 50GHz fixed frequency grid [5], while in optical Flex-grid the spectrum is divided into finer Frequency Slots (FSs) (e.g. $25 \mathrm{GHz}, 12.5 \mathrm{GHz}$ or even $6.25 \mathrm{GHz}$ ) [6][7]. The number of contiguous FSs needed to establish a connection depends on the bitrate demanded the modulation and the frequency grid used [4]. Accordingly,
Flex-grid allows effective spectrum deployment and enables traffic grooming to be performed on the optical layer, instead of demanding an expensive IP/MPLS layer to perform this functionality.

Optical DWDM networks operate within a rigid frequency grid using single-line rate transponders, and apply single carrier modulation techniques. However, the development of single carrier modulation - e.g. QPSK, 16-ary (16-QAM) - i $\mathrm{n}$ mixed line rate networks, added to the advent of multi carrier modulated techniques like OFDM and the flexible usage of the spectral resources in a flexible frequency grids, have made the advent of future optical flex-grid networks possible, whereas it is able to utilise the spectral resources efficiently [3][4][6][8][9]. In addition to these significant advances in optical multi-level modulation techniques, the invention of Band-width Variable Transponders (BVTs) and flexi-grid Reconfigurable Optical Add Drop Multiplexer (ROADM), has enabled the advent of the new Elastic Optical Networks $(\mathrm{EON})$ paradigm [2][3][10].

Several demonstrations of bitrate-variable transmitters have been carried out, to achieve the required bitrate and spectral effectivity. During these demonstrations, the amount of subcarriers or the modulation format has been altered [2] [11]. Yet, if a high-speed BVT is working at lower rate than the maximum, an amount of the BW capacity is unused, therefore Sliceable BVT (SBVT) was invented [14]. A SBVT is capable of assigning its capacity into single or various optical connections, which can then be conveyed to the same or diverse termini [5]. Accordingly, SBVT can be used for exploiting the residual capacity in any channel, if the channel was generated at a low bit rate. Yet, in this architecture SBVT can be regarded either as a high-capacity BVT or as a collection of multiple logically/virtually, depending on the operation mode [10]. Therefore, SBVT has attracted great attention as a new way to use optical networks resources efficiently through facilitating traffic grooming/aggregation directly on the optical layer [12] [13] [14].

It is well known that most of the client's applications are running nowadays on the IP layer. The last 20 years have seen no big changes in the IP layer architecture. ROADM routers are connected to each other via a fixed wavelength grid using discrete transceivers and connected via fixed bitrate links, while the connectivity in the IP topology can be increased by router bypassing using ROADMs. And for efficient utilisation of high-speed wavelength in DWDM networks, IP/MPLS over DWDM multi-layer routing has been introduced. However, this approach is not necessarily cheap, because of the added electrical layer, which will not be the case in the next network generation [10]. 
Initially, concerning its density was the decoupling of the IP layer from the capacity maintained by the transceiver was one of the main triggers for proposing the sliced transponders. SBVT can be divided to cope with the capacity needed to form multiple independent connections between routers. If the available capacity offered by an SBVT is more than the requested capacity, only a slice of the SBVT is assigned to that connection, and the residual capacity can be used for establishing other connections to other destinations. However, we still need to connect the IP/MPLS layer to the optical layer, but approaching the same manner used for fixed transponders, where a single point-to-point connection with fixed capacity is used, will make flexible slicing of a transponder to diverse destinations useless [10]. Consequently, the IP/MPLS layer and optical layer should be interconnected in a different manner. However, the authors in [10] have proposed interconnection options, which use fixed rate interfaces to interconnect the IP/MPLS layer with SBVT in the optical layer. With the purpose of achieving the desired flexibility and attaining the requested bitrate, the SBVT can combine the necessary number of these interfaces into a single light path. This functionality is performed via TDM multiplexing.

Nevertheless, to benefit from such unique flexibility at the optical layer and to utilise the great potential of EONs, the control plane plays a key role.

Currently, most of the automated control planes which have been used for the commercial application of optical transport plans are based on GMPLS protocol suit architecture. GMPLS was standardised by the Internet Engineering Task Force (IETF). The GMPLS protocol suit encompasses the following fundamental protocols. Resource reSerVation Protocol - Traffic Engineering (RSVP-TE), which is responsible for setting up end-to-end quality-enabled connections. Open Shortest Path First - Traffic Engineering (OSPFTE), the duty of which is to distribute topology routing information and traffic engineering (TE) and the creation and maintenance of a TE database (TEDB), to enable the routing at each node in the network. Finally, there is Link Management Protocol (LMP), which has the responsibility of managing the functionality of the links and control channels, and monitoring the connectivity between neighbouring nodes, to help in detecting failures [15].

\section{RELATED WORK AND CONTRIBUTION}

Elastic optical networks have recently received more attention, with several studies investigating their performance and possible utilisation.

The authors of [16] have proposed three patterns (Fixed, Semi-elastic and Elastic) to allocate the variable spectrum in EON. They compared the performances of these patterns in a multi-hour traffic profile. The authors concluded that in a multi-hour traffic scenario, a significant improvement is achieved in the network throughput in comparison to fixed spectrum allocation. Nevertheless, the aspect of multilayer routing using IP/MPLS over EON was not investigated in this research work.

In [17], a flex-grid-based national network is suggested, based on a two-stage procedure. The suggested network consists of a two-stage procedure to design flex-grid based national networks. The suggested network consists of several metro areas, which are interconnected via a flex-grid optical core. The metro areas traffic will be aggregated and conveyed through the optical network, whereas IP/MPLS routers have been used exclusively as metro area edge routers. This study evaluated the spectral efficiency in the function of the metro areas count. However, it did not evaluate the performance of IP/MPLS bases flex-grid optical core within the metro areas, as the authors investigated the performance of flex-grid optical core in long-haul inter-metropolitan links. Furthermore, the authors did not illustrate the efficiency in terms of blocking probability compared to IP/MPLS-based DWDM optical networks.

In [18], the authors considered the spectrum dynamic allocation problem in Orthogonal Frequency-Division Multiplexing (OFDM)-based optical networks, which uses enabling technology like BVT to establish connections using the elastic allocation of low-rate subcarriers according to the connection demands. The authors suggested a policy to assign subcarriers to time-varying traffic in a flexible OFDM optical network and presented a comparison between the OFDM network and the traditional fixed-grid WDM network. The results show that the blocking probability has been reduced due to the flexibility of the OFDM. Yet the authors considered only a traditional fixed-grid WDM network; they did not investigate the traffic grooming feature in multilayer IP/MPLS over DWDM optical networks.

In this research paper, we introduce a new routing scheme which addresses the routing and traffic grooming in optical multilayer IP/MPLS over optical EON networks, in which the traffic demand is dynamically generated. Our proposed routing scheme uses the three fundamental protocols of GMPLS RSVP-TE, OSPF-TE and LMP. We have evaluated our proposed routing and traffic grooming scheme by performing simulation experiments, which illustrate the performance of IP/MPLS base EON networks in terms of routing and traffic forwarding efficiency.

\section{SimUlations AND PERFORMANCE ASSESSMENT}

\section{A. Test Network model}

To carry out our simulations, we used the 28-node Europe network topology (Fig. 1), which was created using OMNET++ [19].

Fig. 1 Europe network topology

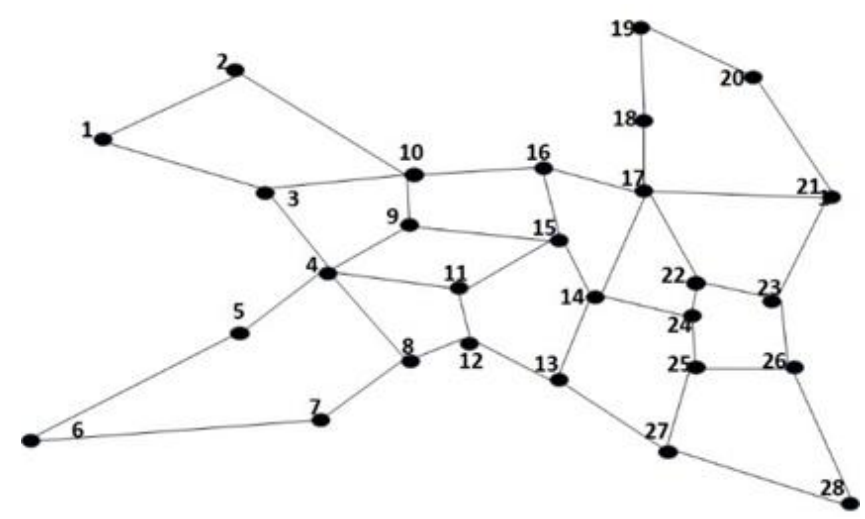

\section{B. Test Node model}

In our test framework we used a simplified node model of incorporated IP/MPLS based optical EON networks, which have the architecture proposed in (Fig. 2) and provides more flexibility in the IP layer topology because of its finer granularity option. 
This architecture uses SBVT-ROADM, which allows the performing of traffic path Label Switching Path (LSP) switching directly on the optical layer, without being forced to execute the label swapping operation in the IP/MPLS router, which makes the electro optical conversion of the light beam superfluous. This architecture makes the node able to perform the following operations:

1. Start/termini point of a Label Switching Path (LSP).

2. Switching point of an OLSP "Lightpath"

3. Switching point of Label Switching Path (LSP).

In switching cases mentioned above in 2 and 3, the traffic being carried over an optical beam using specific wavelength gets into the node through an input fibre, switched to another wavelength and forwarded into an output fibre without any involvement of the IP/MPLS router.

According to capacity needed for a new LSP connection, SBVT slices Bandwidth of each established lightpath connection, which makes creating multiple independent LSP between routers using the same lightpath "OLSP" became applicable, only a slice of the SBVT is assigned to requested connection, while the residual capacity can be used for other LSP connections. This makes it possible to perform subwavelength traffic grooming over high-speed wavelengths on the optical layer.

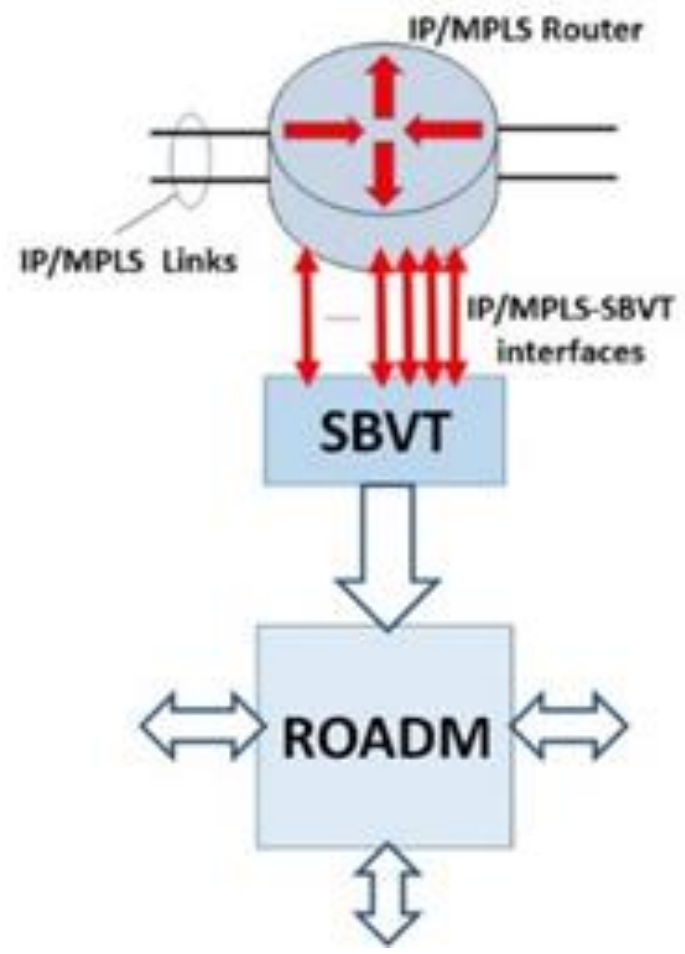

Fig. 2 Integrated IP/MPLS based optical Flexgrid node model

\section{Traffic model}

In the traffic scenarios to this research, the requests for connections are generated by a generator, which selects arbitrarily the source and destination of a connection using a uniform distribution, where traffic initiated in the network is considered as the sum of all the requests for connection. Consequently, each OLSP created has $10 \mathrm{~Gb} / \mathrm{s}$ of Capacity C, while the requested capacity for each connection is $\mathrm{C}_{\text {req }}$, which is arbitrarily selected via a uniform distribution between 10 and $30 \%$ of the OLSP capacity. To determine the connection blocking ratio, we carry out the simulations with $10^{6}$ requests for connection. To evaluate the system performance under various load conditions, the traffic load is increased constantly stepwise during the whole simulation period. Accordingly, the connection request inter-arrival time $T_{i a}$ was fixed to $0.067 \mathrm{~s}$, while the connection holding time $\mathrm{T}_{\mathrm{ht}}$ is increased from 100s min time to 300 s max time.

\section{Applied routing methodology}

In IP/MPLS based EON optical networks, as mentioned above, for each generated source-destination (s-d) pair request for connection, the requested capacity $\mathrm{C}_{\text {req }}$ is known, and we also assume knowledge of the actual traffic rate (e.g. the average rate between each s-d in the network on the optical layer), and the available subcarriers over all the links comprising a path. Accordingly, for each s-d pair the connection path is calculated using the OSPF-TE algorithm, which promises the best utilisation of the available capacity by aggregating subwavelength directly on the optical plane.

\section{E. Routing and connection setup Scheme}

An LSP connection establishing is performed using the of RSVP-TE Loose Route (LR) feature, which is responsible for setting up end-to-end quality-enabled connections [15][20]. The route calculation begins at the originating node and is performed using OSPF-TE routing algorithm (Fig. 3).

As shown in Fig. 3, the originating node calculates the



Fig. 3 Loose Route (LR) calculation using RSVP-TE

end-to-end LR towards the end node, which encompasses the sequence of nodes representing the route to the destination node. The destination node reserves the LSP route based on the wavelength or capacity availability and sends back the RSVP message, including the Explicate Route (ER). Afterwards, the LR originates at source node can be created.

In our simulations, we suggest that all nodes are equipped with wavelength conversion in our test framework.

\section{SimUlation RESUltS}

The performance evaluations of the suggested and applied routing schemes have been done using a request for the connection blocking ratio, which has been performed by diverse traffic load, various wavelength numbers $(16 \lambda, 32 \lambda$, $64 \lambda$ ), and several counts of Optical Electronic Optical (OEO) interfaces, which are responsible for electro optical conversion and hence serve the connection between IP/MPLS client layer and the EON optical layer. 
Fig. 4 reports the blocking ratio measured with 16,32 and $64 \lambda$; it clarifies the effect of wavelength availability on the request for connection blocking probability, which is as predict the higher request for connection blocking ratio if less wavelength is available. This proves the accuracy of our simulation framework and hence our results.

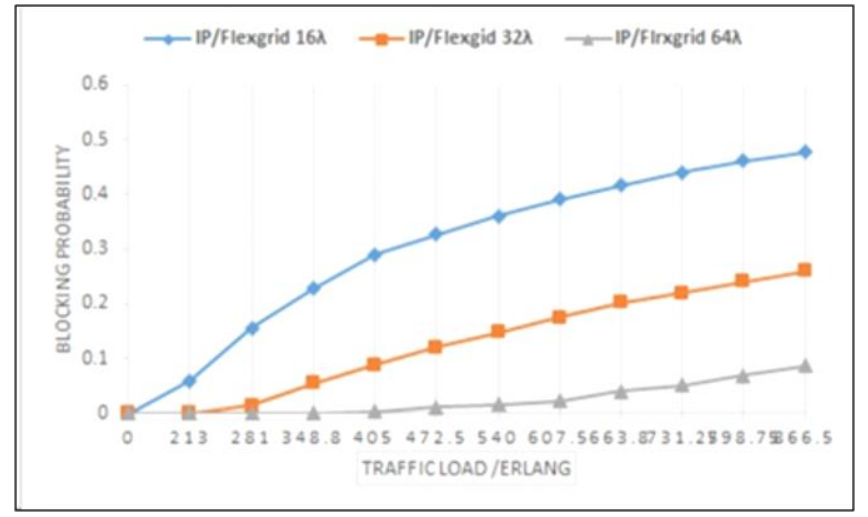

Fig. 4 Blocking ratio by different number of $\lambda$ in function of traffic load

Fig. 5 highlights the impact of the number of OEO interfaces interconnecting the IP/MPLS layer with the SBVTs in an IP/MPLS-based optical EON network on the connection blocking ratio. The results show only a slight difference in the blocking ratio in IP/MPLS over the optical EON network. Moreover, the simulation shows the same behaviour for the $32,64,80$ interfaces till a traffic load of 348.8 Erlang, while the network with 80 interfaces shows a bit less blocking ratio at higher traffic load. The explanation for this behaviour is that the interfaces in IP/MPLS-based EON optical networks are needed only at the LSP connection start and terminus nodes, while the traffic grooming/aggregation is directly performed in the optical layer, without any need to be passed through the interfaces connecting IP/MPLS layer with the optical layer, which is required in IP/MPLS-based rigid DWDM networks.

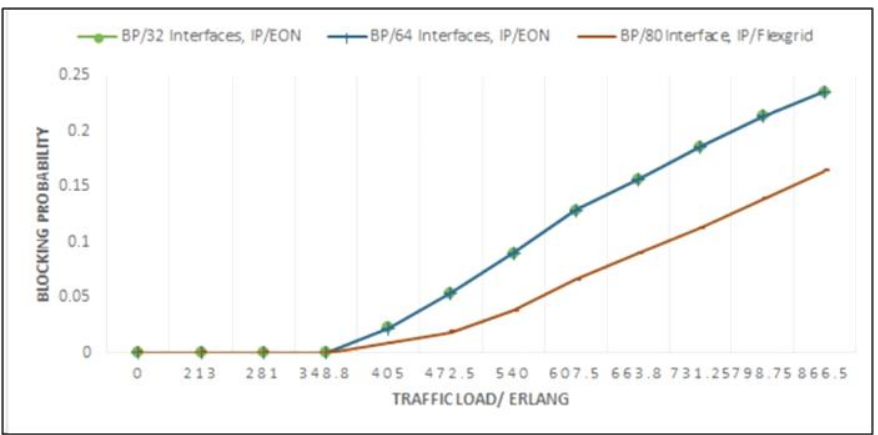

Fig. 5 Blocking ratio by 32, 64, 80 Interfaces in function of traffic load5

\section{CONCLUSIONS AND FUTURE WORK}

In this research paper, we proposed and evaluated a multilayer routing scheme for IP/MPLS-based Elastic Optical Networks (EON).

Our proposed multilayer routing scheme is based on a protocol suite defined by the GMPLS architecture, including RESVP-TE, OSPF-TE and LMP.
To evaluate our proposed routing scheme, a simulation framework for multi-layer optical LSP provisioning was created.

The evaluation of the proposed routing scheme in IP/MPLS-based optical EON has been done in terms of the connection request blocking probability.

The results derived from the simulations show that our proposed routing and connection setup scheme for IP/MPLSbased EON networks offers a good solution for routing and establishing connection setup in IP/MPLS-based EON multilayer networks, which will help in developing a network infrastructure that can cope with the expected growth of global IP traffic, which by 2022 will reach an annual run rate of 4.8 zettabytes per year [1]

The problems of wavelength, Frequency Slot (FS) assignment and spectrum modulation and allocation were not addressed in our research. This can be done in future research work, which requires the upgrade of our proposed routing methodology.

\section{REFERENCES}

[1] Cisco VNI Global IP Traffic Forecast, 2017-2022, https://newsroom.cisco.com/press-releasecontent?type $=$ webcontent\&articleId $=1955935$

[2] O. Gerstel, M. Jinno, A. Lord and S. J. B. Yoo, "Elastic optical networking: a new dawn for the optical layer?" in IEEE Communications Magazine, vol. 50, no. 2, pp. 12-20, February (2012), doi: 10.1109/MCOM.2012.6146481.

[3] M. Jinno, H. Takara, B. Kozicki, Y. Tsukishima, Y. Sone and S. Matsuoka, "Spectrum-Efficient and Scalable Elastic Optical Path Network: Architecture, Benefits, and Enabling Technologies", IEEE Communications Magazine, Vol. 47, pp. 66-73, (2009).

[4] M. Jinno, B. Kozicki, H. Takara, A. Watanabe, Y. Sone, T. Tanaka and A. Hirano, "Distance-adaptive spectrum resource allocation in spectrum-sliced elastic optical path (SLICE) network," IEEE Commun. Mag., vol. 48, pp. 138-145, (2010).

[5] ITU-T G.694.1, "Spectral grids for WDM applications: DWDM frequency grid," May 2002.

[6] Velasco, L., Ruiz, M., Castro, A., Pedrola, O., Klinkowski, M., Careglio, D. and Comellas. On the Performance of Flexgrid-based Optical Networks. 1-4, J. (2013).

[7] Velasco, L., Wright, P., Lord, A. and Junyent, Designing National IP / MPLS Networks with Flexgrid Optical Technology. 1-3, G. (2012).

[8] W. Shieh et al., "Transmission experiment of multi-gigabit coherent optical OFDM systems over $1000 \mathrm{~km}$ SSMF fiber", Electron. Lett., (2007).

[9] J. Armstrong, "OFDM for Optical Communications", IEEE/OSA J. Ligthw. Technol. (2009).

[10] Fernández-@alacios, J. P., López, V., Cruz, B. De, Telefónica, I. D., Sambo, N., Riccardi, E. and Italia, T. (n.d.). Chapter 1 Sliceable Bandwidth Variable Transponders.

[11] M. Jinno, B. Kozicki, H. Takara, A. Watanabe, Y. Sone, T. Tanaka and A. Hirano, "Distance-adaptive spectrum resource allocation in spectrum-sliced elastic optical path (SLICE) network," IEEE Commun. Mag., vol. 48, pp. 138-145, (2010).

[12] L. Velasco, O. Gonzalez de Dios, V. Lopez, J. P. Fernandez-Palacios and G. Junyent: Finding an Objective Cost for Sliceable Flexgrid Transponders, in Proc. Optical Fiber Conference (OFC), Th1E.3, (2014).

[13] B. de la Cruz Miranda, O. Gonzalez de Dios, V. Lopez and J. P. Fernandez-Palacios: OpEx savings by reduction of stock of spare parts with Sliceable Bandwidth Variable Transponders, in Proc. Optical Fiber Conference (OFC), W3A.5, (2014).

[14] Yi, Pan and Ding, Hui and Ramamurthy, Byrav. Cost-Optimized Joint Resource Allocation in Grids/Clouds With Multilayer Optical Network Architecture. Journal of Optical Communications and Networking. 6. 10.1364/JOCN.6.000911, (2014). 
[15] Víctor López, Rodrigo Jiménez, Óscar González de Dios, and Juan Pedro Fernández-Palacios, "Control Plane Architectures for Elastic Optical Networks [Invited]," J. Opt. Commun. Netw. 10, A241-A249 (2018)

[16] L. Velasco, M. Klinkowski, M. Ruiz, V. López and G. Junyent, "Elastic Spectrum Allocation for Variable Traffic in Flexible-Grid Optical Networks," in Optical Fiber Communication Conference, OSA Technical Digest (Optical Society of America, 2012), paper JTh2A.39.

[17] J. Berthold et al., "Optical networking: Past, present, and future," IEEE/OSA J. Lightw. Technol. (2008).
[18] K. Christodoulopoulos, I. Tomkos, and E. Varvarigos, "Dynamic Bandwidth Allocation in Flexible OFDM-based Networks," in Optical Fiber Communication Conference/National Fiber Optic Engineers Conference 2011, OSA Technical Digest (CD) (Optical Society of America, 2011), paper OTuI5.

[19] OMNET++ version 4.0 user manual. http://www.omnetpp.org/ doc/manual/usman.html

[20] L. Berger - "Generalized Multi-Protocol Label Switching (GMPLS) Signaling Resource ReserVation Protocol-Traffic Engineering (RSVPTE) Extensions" - IETF RFC 3473, January 2003.

\section{Creative Commons Attribution License 4.0 (Attribution 4.0 International, CC BY 4.0)}

This article is published under the terms of the Creative Commons Attribution License 4.0

https://creativecommons.org/licenses/by/4.0/deed.en_US 